\title{
Lighting and Light Sources
}

AMNG the papers presented in Section 6 of the International Electrical Congress, recently held in Paris, was one by Dr. Pirani, of the Osram Gesellschaft, who gave a very interesting account of recent developments in the production of light. After a general description of the underlying principles of the modern discharge tube, he dealt in more detail with the two types showing the greatest efficiency, namely, the sodium tube and the mercury tube. In the case of the former it has been found experimentally that the addition of one of the rare gases at a pressure about a thousand times as great as that of the sodium vapour is of considerable advantage. Since the atoms of the rare gas require a very high voltage to excite them, they act principally as elastic obstacles in the path of the electrons and so increase a hundred-fold the distance which an electron travels on its journey from the cathode to the anode. The chance of a collision between the electron and an atom of sodium is thus correspondingly increased. When the tube is operating under the most favourable conditions, 70-80 per cent of the energy of the electrons is transformed into light. An over-all efficiency of about sixty lumens a watt can be obtained from a sodium tube.

In the case of the mercury tube, the intensities of the lines in the longer wave-length part of the spectrum are much increased, relatively to the others, by increasing the pressure in the tube to one atmosphere or more. This is illustrated by diagrams showing the relative intensities of the different lines for two tubes operating at pressures of $0.5 \mathrm{~mm}$. and 3 atmospheres respectively. The result is naturally a very great increase in luminous efficiency and lamps can be operated to give 50 lumens a watt. The possibilities of light production by the excitation of gases in the molecular state, and of liquids or solids, are discussed by the author in a final section of his paper, but he concludes that the most hopeful line of work lies with gases in the atomic state or with vapours.

A new type of mercury arc in quartz, stated to have an efficiency of some 10 candles a watt, was described in a paper by Prof. Cz. Reczynski of Poland. It depends on the reduction of the cathode potential drop from 100 volts to about 5 volts by $(a)$ using for the cathode a ball of tungsten instead of mercury, and $(b)$ placing a tungsten filament at a short distance away from the cathode.

In an interesting communication from Japan, Dr. R. Kurosawa described a method of studying the characteristics of a diffusing material, as regards transmission and reflection. The brightness (not the candle-power) of a sample of the material is measured at all angles of view, the light being incident normally.
The measurements are most conveniently carried out by means of a photoelectric cell (that employed by the author is a vacuum type cæsium cell), an image of a given area of the surface of the material being formed on the sensitive surface of the cell. The author recommends Halbertsma's definition of diffusing power $(\sigma)$, that is,

$$
\sigma=\Sigma^{n} \beta(\theta) / n \beta(o)
$$

where $\beta(\theta)$ is the brightness at an angle $\theta$ to the normal and $n$ is the number of directions in which measurements are made. The author considers that three measurements, at the angles $22 \frac{1}{2}^{\circ}, 45^{\circ}$, and $671^{\circ}$, are generally sufficient. Since $\beta(0)$ cannot be obtained by direct measurement in the case of reflection, it must be deduced by extrapolation from observations made at the above three angles, using Lagrange's formula $\beta(0)=\sum^{3} R_{i} \beta\left(\frac{i \pi}{8}\right)$ where $R_{1}=1.5412, R_{2}=$ $-0.6682, R_{3}=0.1270$. A nomogram is given in the paper for finding $\sigma$ from the observed values of $\beta_{2} / \beta_{1}$ and $\beta_{3} / \beta_{1}$, using the formula deduced by the above method, namely,

$$
\sigma=\left(\beta_{1}+\beta_{2}+\beta_{3}\right) /\left(4 \cdot 62 \beta_{1}-2 \cdot 00 \beta_{2}+0 \cdot 38 \beta_{3}\right) .
$$

The subject of glare in lighting installations was considered in two papers presented in this section. M. J. Dourgnon, defining glare as the threshold sensitivity of the retina at a given point $A$ (for example, the fovea) with the eye exposed to any given field, proposed that the glare produced by a uniform field having a brightness equal to 1 candle per square $\mathrm{cm}$. should be adopted as the 'unit of glare'.

Ing. J. Ondracek, of Austria, dealt principally with the time required for the eye to adapt itself when the gaze was transferred from a surface of high brightness to one of low brightness, or vice versa. This time is a function of the average brightness of the two fields of view and may be expressed in terms of the ratio of these two brightnesses. In the practical case, one brightness is that of the surface being worked upon, while the other is the average brightness of the surroundings, for example, ceiling, walls, and floor.

The use of photoelectric cells for colorimetry was described in a paper by Dr. N. R. Campbell, who outlined the method of colour-matching electric lamps by the use of two cells having markedly different sensitivity curves, and the use of the Toussaint photoelectric colorimeter for giving a rough indication of the spectral distribution of the light reflected from a coloured surface.

\section{Evaporation, Condensation, and Adsorption}

DR. I. LANGMUIR ( $J$. Amer. Chem. Soc., July) has extended his well-known adsorption formula by taking account of the forces between adjacent adsorbed particles ('adatoms'). In the simple theory, the rate of evaporation of adatoms from a surface is $\nu_{1} \theta$, where $\theta$ is the fraction of the surface covered and $v_{1}$ a constant. If atoms condense only on uncovered. parts, the rate of condensation is $\alpha_{0} \mu(1-\theta)$, where $\alpha_{0}$ is constant, measuring the efficiency of condensation on a bare surface, and $\mu$ the rate of collision of incident atoms per unit area of surface, given by the kinetic theory :

$$
\mu=(2 \pi m k T)^{-\frac{1}{2}} p=2.653 \times 10^{19} p(M T)^{-\frac{1}{2}} .
$$

where $p=$ pressure in baryes, $k=$ Boltzmann's conNo. 3283, VoL. 130] stant, $m$ is the mass of an atom, and $M$ the atomic weight of the gas. For steady states the rates of evaporation and condensation are equal, hence

$$
\theta=\alpha_{0} \mu /\left(\nu_{1}+\alpha_{0} \mu\right)=\alpha_{0} \tau \mu /\left(\sigma_{1}+\alpha_{0} \tau \mu\right)
$$

where $\tau=\sigma_{1} / \nu_{1}=$ average life of an adatom, $\sigma_{1}$ being the number of adatoms per unit area of saturated surface $(\theta=1)$. Equation (2) represents the observed adsorption on plane surfaces with reasonable accuracy in a surprisingly large number of cases.

The vapour pressure of a liquid is given over a wide range of temperature by

$$
p=A T^{\gamma} e^{-b i T} \simeq A_{\theta} e^{-b_{0} / T} .
$$

Trouton's rule requires that $\gamma=0$ and $A$ is a universal 